\title{
ASYMPTOTIC ANALYSIS OF A HERMITIAN MATRIX INTEGRAL*
}

\author{
BY \\ Motohico Mulase** \\ Department of Mathematics \\ University of California, Davis \\ Davis, CA 95616 \\ mulase@math.ucdavis.edu
}

\begin{abstract}
The asymptotic expansion of a Hermitian matrix integral known as the Penner model is rigorously calculated.
\end{abstract}

\section{Introduction.}

The purpose of this paper is to establish an asymptotic analysis of a Hermitian matrix integral known as the Penner model, and to calculate its asymptotic expansion. It was proved by Penner [7] that this asymptotic series gives the orbifold Euler characteristic of the moduli spaces of pointed algebraic curves. The formula he obtained is in agreement with the result of Harer and Zagier [2].

Both Harer-Zagier [2] and Penner [7] use a Hermitian matrix integral to compute the Euler characteristic $\chi\left(\mathfrak{M}_{g, s}\right)$ of the moduli space $\mathfrak{M}_{g, s}$ of stable algebraic curves of genus $g$ with $s$ smooth marked points. Among the two, Penner's method is conceptually easier to understand. He computes the asymptotic expansion of the Hermitian matrix integral in two different ways: one by using the Feynman diagram expansion, and the other by direct computation using analytic continuation. Since the asymptotic expansion is unique, the two methods should give the same answer. Penner [7] established, based on the work of 't Hooft [8] and Bessis-Itzykson-Zuber [1], that the Feynman diagram expansion of the matrix integral gives a generating function of $\chi\left(\mathfrak{M}_{g, s}\right)$ for $g \geq 0$ and $s>0$ with $2-2 g-s<0$. He then computed the matrix integral by using analytic continuation and obtained the expression of the rational numbers $\chi\left(\mathfrak{M}_{g, s}\right)$ in terms of the special value $\zeta(1-2 g)$ of the Riemann

1991 Mathematics Subject Classification. Primary: 81C30, 30E15, 30E20, 33A65, $14 \mathrm{H} 15$.

*Published in International Journal of Mathematics 6 (1995) 881-892.

**Research supported in part by NSF Grant DMS 94-04111. 
zeta-function. However, the formula he suggested to compute the matrix integral does not seem to hold in the holomorphic category.

We show in this paper that Penner's formula is true in the asymptotic category, which is sufficient to give the asymptotic expansion of the Penner model. Once we establish a rigorous asymptotic analysis, Penner's method of computation of the Euler characteristic works without any major modification.

The contribution of this paper is Theorem 4.2, which replaces a corresponding analytic statement found in Penner's paper. The formula we establish does not hold in the analytic category. With this Theorem, computation of the asymptotic expansion of the Penner model becomes more straightforward and easier.

\section{The Feynman diagram expansion of the Hermitian matrix integral.}

Let us begin with recalling the asymptotic expansion of a holomorphic function. Let $\Omega$ be an open domain of the complex plane $\mathbb{C}$ having the origin 0 on its boundary, and let $h(z)$ be a holomorphic function defined on $\Omega$. A formal power series

$$
\sum_{\nu=0}^{\infty} a_{\nu} z^{\nu}
$$

is said to be an asymptotic expansion of $h(z)$ on $\Omega$ at $z=0$ if

$$
\lim _{\substack{z \rightarrow 0 \\ z \in \Omega}} \frac{h(z)-\sum_{\nu=0}^{m} a_{\nu} z^{\nu}}{z^{m+1}}=a_{m+1}
$$

holds for all $m \geq 0$. The above formula shows that if $h(z)$ admits an asymptotic expansion, then it is unique. However, one cannot recover the original holomorphic function from its asymptotic expansion. A simple example is $e^{-1 / z}$ defined on the left-half plane $\{z \in \mathbb{C} \mid \operatorname{Re}(z)<0\}$, whose asymptotic expansion at the origin is the 0 -series. Sometimes this fact works positively: we will use it to simplify the matrix integral in Section 4. Since the asymptotic expansion of a holomorphic function is not equal to the original function, we use the following notation to indicate that (2.1) is the asymptotic expansion of a holomorphic function $h(z)$ :

$$
\mathcal{A}(h(z))=\sum_{\nu=0}^{\infty} a_{\nu} z^{\nu}
$$

If two holomorphic functions $h(z)$ and $f(z)$ defined on $\Omega$ have the same asymptotic expansion at $z=0$, we use the notation

$$
h(z) \stackrel{A}{\equiv} f(z) .
$$


Thus $0 \stackrel{A}{\equiv} e^{-1 / z}$ at $z=0$ as holomorphic functions defined on the left-half plane.

The asymptotic expansion also applies to real analytic functions. For example, if $K$ is the positive real axis and $h(z)$ is a real analytic function on $K$, then the same formula (2.2) defines the asymptotic expansion of $h(z)$ at $z=0$.

The matrix integral we deal with in this paper is the following:

$$
Z_{n}(t, m)=\int_{\mathcal{H}_{n}} \exp \left(-\frac{1}{2} \operatorname{trace}\left(X^{2}\right)\right) \exp \left(\operatorname{trace} \sum_{j=3}^{2 m} \frac{t_{j}}{j} X^{j}\right) d X
$$

where $\mathcal{H}_{n}$ is the space of all $n \times n$ Hermitian matrices, and $d X$ denotes the usual Lebesgue measure of $\mathcal{H}_{n}$ as a real vector space of dimension $n^{2}$. We note that $Z_{n}(t, m)$ is a holomorphic function for all values of $\left(t_{3}, t_{4}, \cdots, t_{2 m-1}\right) \in \mathbb{C}^{2 m-3}$ and $t_{2 m}$ satisfying that $R e\left(t_{2 m}\right)<0$. We can expand $Z_{n}(t, m)$ as a convergent power series in $t_{3}, t_{4}, \cdots, t_{2 m-1}$ about 0 , and as an asymptotic series in $t_{2 m}$ as $t_{2 m} \rightarrow 0$ with $\operatorname{Re}\left(t_{2 m}\right)<0$.

It is a well established theorem ([1], [3], [7], [8], and [9]) that the Feynman diagram technique gives the asymptotic expansion

$$
\left.\log \mathcal{A}\left(\frac{Z_{n}(t, m)}{Z_{n}(0)}\right)=\sum_{\substack{g \geq 0, s>0 \\ 2-2 g-s<0}} \sum_{\substack{\text { connected ribbon graph } \Gamma \\ \text { with valency } 3,4, \cdots, 2 m, \chi(\Gamma)=2-2 g-s, s(\Gamma)=s}} \frac{n^{s}}{\# \operatorname{Aut}(\Gamma)} \cdot \prod_{j=3}^{2 m} t_{j}^{v_{j}(\Gamma)}\right)
$$

with respect to $t_{2 m}$, as $t_{2 m} \rightarrow 0$ with $R e\left(t_{2 m}\right)<0$, where a ribbon graph (or fatgraph $\Gamma$ is a 1-dimensional $C W$-complex with cyclic ordering of edges at each vertex, $\chi(\Gamma)$ the Euler characteristic of the graph $\Gamma, s(\Gamma)$ the number of closed loops in $\Gamma, \operatorname{Aut}(\Gamma)$ the automorphism group of the ribbon graph, $v_{j}(\Gamma)$ the number of $j$-valent vertices of $\Gamma$, and

$$
Z_{n}(0)=\int_{\mathcal{H}_{n}} \exp \left(-\frac{1}{2} \operatorname{trace}\left(X^{2}\right)\right) d X .
$$

Let $v(\Gamma)$ and $e(\Gamma)$ be the total number of vertices and edges of the graph $\Gamma$, respectively. Then

$$
\left\{\begin{array}{l}
\chi(\Gamma)=v(\Gamma)-e(\Gamma) \\
v(\Gamma)=v_{3}(\Gamma)+v_{4}(\Gamma)+\cdots+v_{2 m}(\Gamma) \\
e(\Gamma)=\frac{1}{2}\left(3 \cdot v_{3}(\Gamma)+4 \cdot v_{4}(\Gamma)+\cdots+2 m \cdot v_{2 m}(\Gamma)\right)
\end{array}\right.
$$

because $\Gamma$ has valency in between 3 and $2 m$. Thus for every fixed $g$ and $s$, the second summation of (2.4) is a finite sum. It shows that the right-hand-side of (2.4) is an element of the formal power series ring

$$
\mathbb{C}\left[\left[t_{3}, t_{4}, \cdots, t_{2 m}\right]\right]
$$


Let us define the formal power series ring of infinitely many variables $\mathbb{C}\left[\left[t_{3}, t_{4}, t_{5}, \cdots\right]\right]$. The adic topology of this ring is given by the degree

$$
\operatorname{deg} t_{j}=j, \quad j \geq 3
$$

and the ideal $\mathfrak{I}_{j}$ of $\mathbb{C}\left[\left[t_{3}, t_{4}, t_{5}, \cdots\right]\right]$ generated by all the homogeneous polynomials of degree $j$. We have a natural projection

$$
\pi_{j}: \mathbb{C}\left[\left[t_{3}, t_{4}, t_{5}, \cdots\right]\right] \longrightarrow \mathbb{C}\left[\left[t_{3}, t_{4}, t_{5}, \cdots\right]\right] / \mathfrak{I}_{j}
$$

For each fixed $j$, the projection image

$$
\pi_{j}\left(\log \mathcal{A}\left(\frac{Z_{n}(t, m)}{Z_{n}(0)}\right)\right) \in \mathbb{C}\left[\left[t_{3}, t_{4}, t_{5}, \cdots\right]\right] / \mathfrak{I}_{j}
$$

is stable for all $2 m \geq j$. Since

$$
\mathbb{C}\left[\left[t_{3}, t_{4}, t_{5}, \cdots\right]\right]=\lim _{j} \mathbb{C}\left[\left[t_{3}, t_{4}, t_{5}, \cdots\right]\right] / \mathfrak{I}_{j}
$$

and

$$
\left\{\pi_{2 m}\left(\log \mathcal{A}\left(\frac{Z_{n}(t, m)}{Z_{n}(0)}\right)\right)\right\}_{m \geq 2}
$$

defines an element of the projective system, it gives a formal power series in infinitely many variables. We denote the above element (2.6) symbolically by

$$
\lim _{m \rightarrow \infty} \log \mathcal{A}\left(\frac{Z_{n}(t, m)}{Z_{n}(0)}\right)
$$

which is a well-defined formal power series in infinitely many variables.

Going back to the Feynman diagram expansion (2.4), we have an equality

$$
\lim _{m \rightarrow \infty} \log \mathcal{A}\left(\frac{Z_{n}(t, m)}{Z_{n}(0)}\right)=\sum_{\substack{g \geq 0, s>0 \\ 2-2 g-s<0}}\left(\sum_{\substack{\text { connected ribbon graph } \Gamma \\ \text { with valency } \geq 3 \text { and } \\ \chi(\Gamma)=2-2 g-s, s(\Gamma)=s}} \frac{n^{s}}{\# \operatorname{Aut}(\Gamma)} \cdot \prod_{j \geq 3} t_{j}^{v_{j}(\Gamma)}\right)
$$

as a well-defined element of $\mathbb{C}\left[\left[t_{3}, t_{4}, t_{5}, \cdots\right]\right]$. For each fixed $g$ and $s$, the maximum possible valency of the graphs in the second summation is $4 g+2 s-2$. To see this, let $\Gamma$ be a graph with the largest possible valency $\ell$. Since the Euler characteristic of $\Gamma$ is given by $2-2 g-s=v(\Gamma)-e(\Gamma)$, the valency becomes maximum when $\Gamma$ has only one vertex. Thus

$$
2-2 g-s=1-\frac{1}{2} \ell \text {. }
$$

This shows that the right-hand-side of (2.8) does not have any infinite products.

Another interesting fact about the Hermitian matrix integral (2.3) is that it is a solution to the KP equations. We refer to [5] and [6] for more detail about this topic. 


\section{The Penner model.}

Following Penner, let us make a substitution

$$
t_{j}=-(\sqrt{z})^{j-2}, \quad j=3,4,5, \cdots
$$

in $(2.3)$, where $\sqrt{z}$ is defined for $\operatorname{Re}(z)>0$. The condition $\operatorname{Re}\left(t_{2 m}\right)<0$ for $t_{2 m}$ translates into the condition $\operatorname{Re}\left(z^{m-1}\right)>0$, which is satisfied if

$$
|\arg (z)|<\frac{\pi}{2 m-2}
$$

holds. Thus we have a holomorphic function

$$
\begin{aligned}
P_{n}(z, m) & =\frac{1}{Z_{n}(0)} \int_{\mathcal{H}_{n}} \exp \left(-\frac{1}{2} \operatorname{trace}\left(X^{2}\right)\right) \exp \left(-\sum_{j=3}^{2 m} \frac{(\sqrt{z})^{j-2}}{j} \operatorname{trace}\left(X^{j}\right)\right) d X \\
& =\frac{1}{Z_{n}(0)} \int_{\mathcal{H}_{n}} \exp \left(-\sum_{j=2}^{2 m} \frac{(\sqrt{z})^{j-2}}{j} \operatorname{trace}\left(X^{j}\right)\right) d X
\end{aligned}
$$

defined on the region of the complex plane determined by (3.2). Its asymptotic expansion at $z=0$ can be calculated by making the same substitution (3.1) in $(2.4)$ :

$$
\log \mathcal{A}\left(P_{n}(z, m)\right)=\sum_{\substack{g \geq 0, s>0 \\
2-2 g-s<0}}\left(\sum_{\begin{array}{c}
\text { connected ribbon graph } \Gamma \\
\text { with valency } 3,4, \cdots, 2 m, \\
\chi(\Gamma)=2-2 g-s, s(\Gamma)=s
\end{array}} \frac{(-1)^{e(\Gamma)}}{\# \operatorname{Aut}(\Gamma)}\right) n^{s} \cdot(-z)^{2 g+s-2},
$$

where we used (2.5) to compute

$$
\begin{aligned}
\prod_{j=3}^{2 m}\left(-(\sqrt{z})^{j-2}\right)^{v_{j}(\Gamma)} & =(-1)^{\sum_{j=3}^{2 m} v_{j}(\Gamma)} \cdot z^{\frac{1}{2} \sum_{j=3}^{2 m} j v_{j}(\Gamma)-\sum_{j=3}^{2 m} v_{j}(\Gamma)} \\
& =(-1)^{v(\Gamma)} z^{e(\Gamma)-v(\Gamma)} \\
& =(-1)^{e(\Gamma)}(-z)^{-\chi(\Gamma)} .
\end{aligned}
$$

Note that the right-hand-side of $(3.4)$ is a well-defined element of $(\mathbb{C}[n])[[z]]$. For every $\nu>0$, the terms of $\log \mathcal{A}\left(P_{n}(z, m)\right)$ of degree less than or equal to $\nu$ with respect to $z$ are stable for all $m \geq \nu+1$. Again by the same argument we used in Section 2, we can define an element

$$
\lim _{m \rightarrow \infty} \log \mathcal{A}\left(P_{n}(z, m)\right) \in(\mathbb{C}[n])[[z]] .
$$


Thus we have an equality

$$
\begin{aligned}
& \lim _{m \rightarrow \infty} \log \mathcal{A}\left(\frac{1}{Z_{n}(0)} \int_{\mathcal{H}_{n}} \exp \left(-\sum_{j=2}^{2 m} \frac{(\sqrt{z})^{j-2}}{j} \operatorname{trace}\left(X^{j}\right)\right) d X\right) \\
= & \sum_{\substack{g \geq 0, s>0 \\
2-2 g-s<0}}\left(\sum_{\substack{\text { connected ribbon graph } \Gamma \\
\text { with valency } \geq 3, \chi(\Gamma)=2-2 g-s, s(\Gamma)=s}}^{\# \operatorname{Aut}(\Gamma)} n^{s} \cdot(-z)^{2 g+s-2}\right. \\
& \left.\frac{(-1)^{e(\Gamma)}}{\#-2}\right)
\end{aligned}
$$

as a well-defined element of $(\mathbb{C}[n])[[z]]$. It has been established in $[7]$ that

$$
\chi\left(\mathfrak{M}_{g, s}\right)=(-1)^{s} \cdot \sum_{\begin{array}{c}
\text { connected ribbon graph } \Gamma \\
\text { with valency } \geq 3, \\
\chi(\Gamma)=2-2 g-s, s(\Gamma)=s
\end{array}} \frac{(-1)^{e(\Gamma)}}{\# \operatorname{Aut}(\Gamma)}
$$

for $g \geq 0$ and $s>0$ such that $2-2 g-s<0$, where $\mathfrak{M}_{g, s}$ is the moduli space of stable algebraic curves with (unordered) $s$ marked nonsingular points specified. Therefore, the formal power series $\lim _{m \rightarrow \infty} \log \mathcal{A}\left(P_{n}(z, m)\right)$ in $n$ and $z$ gives a generating function of the orbifold Euler characteristic of the moduli spaces of pointed algebraic curves.

\section{The asymptotic analysis.}

Let us now compute $\lim _{m \rightarrow \infty} \log \mathcal{A}\left(P_{n}(z, m)\right)$. The standard analytic technique to compute the Hermitian matrix integrals (2.3) and (3.3) is the following formula:

Formula 4.1. [1], [2], [4]. Let $f(X)$ be a function on $X \in \mathcal{H}_{n}$ which is invariant under the conjugation by a unitary matrix $U \in U(n)$ :

$$
f(X)=f\left(U^{-1} \cdot X \cdot U\right)=f\left(k_{0}, k_{1}, \cdots, k_{n-1}\right),
$$

where $k_{0}, k_{1}, \cdots, k_{n-1}$ are the eigenvalues of the Hermitian matrix $X$. If $f(X)$ is integrable on $\mathcal{H}_{n}$ with respect to the measure $d X$, then

$$
\int_{\mathcal{H}_{n}} f(X) d X=c(n) \cdot \int_{\mathbb{R}^{n}} f\left(k_{0}, k_{1}, \cdots, k_{n-1}\right) \Delta(k)^{2} d k_{0} d k_{1} \cdots d k_{n-1},
$$

where

$$
c(n)=\frac{\pi^{n(n-1) / 2}}{n ! \cdot(n-1) ! \cdots 2 ! \cdot 1 !},
$$


and

$$
\Delta(k)=\Delta\left(k_{0}, k_{1}, \cdots, k_{n-1}\right)=\prod_{i>j}\left(k_{i}-k_{j}\right)=\operatorname{det}\left(k_{i}^{j}\right)
$$

is the Vandermonde determinant.

Thus we have

$$
P_{n}(z, m)=\frac{c(n)}{Z_{n}(0)} \int_{\mathbb{R}^{n}} \Delta(k)^{2} \prod_{i=0}^{n-1}\left(\exp \left(-\sum_{j=2}^{2 m} \frac{(\sqrt{z})^{j-2}}{j} k_{i}^{j}\right) d k_{i}\right) .
$$

The following is our main result.

Theorem 4.2. Let $\mathfrak{I}_{\nu}=z^{\nu} \cdot \mathbb{C}[[z]]$ denote the ideal of $\mathbb{C}[[z]]$ generated by $z^{\nu}$, and

$$
\pi_{\nu}: \mathbb{C}[[z]] \longrightarrow \mathbb{C}[[z]] / \mathfrak{I}_{\nu}
$$

the natural projection. For a polynomial $p(k) \in \mathbb{C}[k]$, consider the following two asymptotic series:

$$
a(z, m)=\mathcal{A}\left(\int_{-\infty}^{\infty} p(k) \cdot \exp \left(-\sum_{j=2}^{2 m} \frac{(\sqrt{z})^{j-2}}{j} k^{j}\right) d k\right) \in \mathbb{C}[[z]]
$$

as $z \rightarrow+0$ with $|\arg (z)|<\frac{\pi}{2 m-2}$, and

$$
b(z)=\mathcal{A}\left(\sqrt{z}(e z)^{1 / z} \int_{0}^{\infty} p\left(\frac{1-z x}{\sqrt{z}}\right) \cdot x^{1 / z} \cdot e^{-x} \cdot d x\right) \in \mathbb{C}[[z]]
$$

as $z \rightarrow+0$ with $z>0$. Then for every $m>2$, we have

$$
\pi_{m}(a(z, m))=\pi_{m}(b(z))
$$

as an element of $\mathbb{C}[[z]] / \mathfrak{I}_{m}$. In other words,

$$
\begin{aligned}
& \lim _{m \rightarrow \infty} \mathcal{A}\left(\int_{-\infty}^{\infty} p(k) \cdot \exp \left(-\sum_{j=2}^{2 m} \frac{(\sqrt{z})^{j-2}}{j} k^{j}\right) d k\right) \\
= & \mathcal{A}\left(\sqrt{z}(e z)^{1 / z} \int_{0}^{\infty} p\left(\frac{1-z x}{\sqrt{z}}\right) \cdot x^{1 / z} \cdot e^{-x} \cdot d x\right)
\end{aligned}
$$

holds with respect to the $\mathfrak{I}_{m}$-adic topology of $\mathbb{C}[[z]]$. 
Proof. Putting $y=\sqrt{z} k$, we have

$$
\begin{aligned}
\int_{-\infty}^{\infty} p(k) \cdot \exp \left(-\sum_{j=2}^{2 m} \frac{(\sqrt{z})^{j-2}}{j} k^{j}\right) d k & =\frac{1}{\sqrt{z}} \int_{-\infty}^{\infty} p\left(\frac{y}{\sqrt{z}}\right) \cdot \exp \left(-\frac{1}{z} \sum_{j=2}^{2 m} \frac{y^{j}}{j}\right) d y \\
& =\int_{-\infty}^{\infty} d \nu(y, m),
\end{aligned}
$$

where

$$
d \nu(y, m)=\frac{1}{\sqrt{z}} \cdot p\left(\frac{y}{\sqrt{z}}\right) \cdot \exp \left(-\frac{1}{z} \sum_{j=2}^{2 m} \frac{y^{j}}{j}\right) d y .
$$

Let us decompose the integral into three pieces:

$$
\int_{-\infty}^{\infty} d \nu(y, m)=\int_{-\infty}^{-1} d \nu(y, m)+\int_{-1}^{1} d \nu(y, m)+\int_{1}^{\infty} d \nu(y, m) .
$$

Note that the polynomial

$$
\sum_{j=2}^{2 m} \frac{y^{j}}{j}
$$

of degree $2 m$ takes positive values on the intervals $(-\infty,-1]$ and $[1, \infty)$. Since $p(k)$ is a polynomial, it is obvious that the asymptotic expansion of the first and the third integrals of the right-hand-side of (4.3) for $z \rightarrow+0$ with $z>0$ are the 0 -series. Therefore, we have

$$
\int_{-\infty}^{\infty} d \nu(y, m) \stackrel{A}{\equiv} \int_{-1}^{1} d \nu(y, m) .
$$

On the interval $[-1,1]$, if we fix a $z$ such that $|\arg (z)|<\pi$, then the convergence

$$
\lim _{m \rightarrow \infty} \exp \left(-\frac{1}{z} \sum_{j=2}^{2 m} \frac{y^{j}}{j}\right)=(1-y)^{1 / z} \cdot e^{y / z}
$$

is absolute and uniform with respect to $y$. Therefore, in a new variable $t=1-y$, we have

$$
\begin{aligned}
\lim _{m \rightarrow \infty} \int_{-1}^{1} d \nu(y, m)= & \frac{1}{\sqrt{z}} \int_{-1}^{1} p\left(\frac{y}{\sqrt{z}}\right)(1-y)^{1 / z} e^{y / z} d y \\
= & \frac{1}{\sqrt{z}} e^{1 / z} \int_{0}^{2} p\left(\frac{1-t}{\sqrt{z}}\right) t^{1 / z} e^{-t / z} d t \\
= & \frac{1}{\sqrt{z}} e^{1 / z} \int_{0}^{\infty} p\left(\frac{1-t}{\sqrt{z}}\right) t^{1 / z} e^{-t / z} d t \\
& -\frac{1}{\sqrt{z}} e^{1 / z} \int_{2}^{\infty} p\left(\frac{1-t}{\sqrt{z}}\right) t^{1 / z} e^{-t / z} d t
\end{aligned}
$$


This last integral is

$$
\frac{1}{\sqrt{z}} e^{1 / z} \int_{2}^{\infty} p\left(\frac{1-t}{\sqrt{z}}\right) t^{1 / z} e^{-t / z} d t=\frac{1}{\sqrt{z}} \int_{2}^{\infty} p\left(\frac{1-t}{\sqrt{z}}\right) e^{(1+\log t-t) / z} d t .
$$

Since $1+\log t-t<0$ for $t \geq 2$, the asymptotic expansion of this integral as $z \rightarrow+0$ with $z>0$ is the 0 -series. Therefore, since the integrals do not depend on the integration variables, we have

$$
\begin{aligned}
& \lim _{m \rightarrow \infty} \mathcal{A}\left(\int_{-\infty}^{\infty} p(k) \cdot \exp \left(-\sum_{j=2}^{2 m} \frac{(\sqrt{z})^{j-2}}{j} k^{j}\right) d k\right) \\
= & \mathcal{A}\left(\frac{1}{\sqrt{z}} e^{1 / z} \int_{0}^{\infty} p\left(\frac{1-t}{\sqrt{z}}\right) t^{1 / z} e^{-t / z} d t\right) \\
= & \mathcal{A}\left(\sqrt{z} e^{1 / z} z^{1 / z} \int_{0}^{\infty} p\left(\frac{1-z x}{\sqrt{z}}\right) x^{1 / z} e^{-x} d x\right)
\end{aligned}
$$

as a formal power series in $z$. This completes the proof of Theorem.

By applying Theorem 4.2 for each $k_{i}$, we obtain

$$
\begin{aligned}
& \lim _{m \rightarrow \infty} \mathcal{A}\left(\int_{\mathbb{R}^{n}} \Delta(k)^{2} \cdot \prod_{i=0}^{n-1} \exp \left(-\sum_{j=2}^{2 m} \frac{(\sqrt{z})^{j-2}}{j} k_{i}^{j}\right) d k_{i}\right) \\
= & \mathcal{A}\left(\left(\sqrt{z} e^{1 / z} z^{1 / z}\right)^{n} \int_{0}^{\infty} \cdots \int_{0}^{\infty} \Delta\left(\frac{1-z x}{\sqrt{z}}\right)^{2} \cdot \prod_{i=0}^{n-1} x_{i}^{1 / z} e^{-x_{i}} d x_{i}\right) \\
= & \mathcal{A}\left(\left(\sqrt{z} e^{1 / z} z^{1 / z}\right)^{n} z^{\frac{n(n-1)}{2}} \int_{0}^{\infty} \cdots \int_{0}^{\infty} \Delta(x)^{2} \cdot \prod_{i=0}^{n-1} x_{i}^{1 / z} e^{-x_{i}} d x_{i}\right) .
\end{aligned}
$$

We can use the standard technique of orthogonal polynomials for the above integral. Let $p_{j}(x)$ be a monic orthogonal polynomial in $x$ of degree $j$ with respect to the measure

$$
d \mu(x)=x^{1 / z} e^{-x} d x
$$

defined on $K=(0, \infty]$ for a positive $z>0$ :

$$
\int_{K} p_{i}(x) p_{j}(x) d \mu(x)=\delta_{i j}\left\|p_{j}(x)\right\|^{2} .
$$

Because of the multilinearity of the determinant, we have

$$
\Delta(x)=\operatorname{det}\left(x_{i}^{j}\right)_{9}=\operatorname{det}\left(p_{j}\left(x_{i}\right)\right) .
$$


Therefore,

$$
\begin{aligned}
\int_{K^{n}} \Delta(x)^{2} d \mu\left(x_{0}\right) \cdots d \mu\left(x_{n-1}\right) & =\int_{K^{n}} \operatorname{det}\left(p_{j}\left(x_{i}\right)\right) \operatorname{det}\left(p_{j}\left(x_{i}\right) d \mu\left(x_{i}\right)\right) \\
& =\int_{K^{n}} \sum_{\sigma \in S_{n}} \sum_{\tau \in S_{n}} \prod_{i=0}^{n-1} p_{\sigma(i)}\left(x_{i}\right) \prod_{i=0}^{n-1} p_{\tau(i)}\left(x_{i}\right) d \mu\left(x_{i}\right) \\
& =\sum_{\sigma \in S_{n}} \sum_{\tau \in S_{n}} \prod_{i=0}^{n-1} \int_{K} p_{\sigma(i)}(x) p_{\tau(i)}(x) d \mu(x) \\
& =\sum_{\sigma \in S_{n}} \prod_{i=0}^{n-1} \int_{K} p_{\sigma(i)}(x) p_{\sigma(i)}(x) d \mu(x) \\
& =n ! \prod_{i=0}^{n-1}\left\|p_{i}(x)\right\|^{2} .
\end{aligned}
$$

For a real number $z>0$, the Laguerre polynomial

$$
L_{m}^{1 / z}(x)=\sum_{j=0}^{m}\left(\begin{array}{c}
m+1 / z \\
m-j
\end{array}\right) \frac{(-1)^{j}}{j !} x^{j}=\frac{(-1)^{m}}{m !} x^{m}+\cdots
$$

of degree $m$ satisfies the orthogonality condition

$$
\int_{0}^{\infty} L_{i}^{1 / z}(x) L_{j}^{1 / z}(x) e^{-x} x^{1 / z}=\delta_{i j} \frac{(j+1 / z) !}{j !} .
$$

Thus we can use

$$
p_{i}(x)=(-1)^{i} \cdot i ! \cdot L_{i}^{1 / z}(x)
$$

for the computation. From (4.4)-(4.7), we have

$$
\begin{aligned}
& \lim _{m \rightarrow \infty} \mathcal{A}\left(\int_{\mathbb{R}^{n}} \Delta(k)^{2} \cdot \prod_{i=0}^{n-1} \exp \left(-\sum_{j=2}^{2 m} \frac{(\sqrt{z})^{j-2}}{j} k_{i}^{j}\right) d k_{i}\right) \\
= & \mathcal{A}\left(\left(\sqrt{z} e^{1 / z} z^{1 / z}\right)^{n} z^{\frac{n(n-1)}{2}} n ! \prod_{i=0}^{n-1} i ! \cdot\left(i+\frac{1}{z}\right) !\right) \\
= & \mathcal{A}\left((e z)^{\frac{n}{z}} \cdot z^{\frac{n^{2}}{2}} \cdot n ! \prod_{i=0}^{n-1} i ! \cdot\left(-1+\frac{1}{z}\right) ! \cdot\left(i+\frac{1}{z}\right)^{n-i}\right) .
\end{aligned}
$$


Applying Formula 4.1 and (4.8) to (3.5), we conclude

$$
\begin{aligned}
& \lim _{m \rightarrow \infty} \log \mathcal{A}\left(\frac{1}{Z_{n}(0)} \int_{\mathcal{H}_{n}} \exp \left(-\sum_{j=2}^{2 m} \frac{(\sqrt{z})^{j-2}}{j} \operatorname{trace}\left(X^{j}\right)\right) d X\right) \\
= & \log \mathcal{A}\left(\frac{1}{Z_{n}(0)} \cdot \pi^{\frac{n(n-1)}{2}} \cdot(e z)^{\frac{n}{z}} \cdot z^{\frac{n^{2}}{2}} \cdot \prod_{i=0}^{n-1}\left(-1+\frac{1}{z}\right) ! \cdot\left(i+\frac{1}{z}\right)^{n-i}\right) \\
= & \log \mathcal{A}\left(\frac{1}{Z_{n}(0)} \cdot \pi^{\frac{n(n-1)}{2}} \cdot(e z)^{\frac{n}{z}} \cdot z^{\frac{n^{2}}{2}} \cdot\left(\Gamma\left(\frac{1}{z}\right)\right)^{n} \cdot \prod_{i=0}^{n-1}\left(i+\frac{1}{z}\right)^{n-i}\right) \\
= & \operatorname{const}+\frac{n}{z}+\frac{n}{z} \log z+\frac{n^{2}}{2} \log z+n \log \mathcal{A}\left(\Gamma\left(\frac{1}{z}\right)\right)+\sum_{i=0}^{n-1}(n-i) \log \frac{1+i z}{z} \\
= & \operatorname{const}+\frac{n}{z}+\frac{n}{z} \log z-\frac{n}{2} \log z+n \log \mathcal{A}\left(\Gamma\left(\frac{1}{z}\right)\right) \\
& +\sum_{r=1}^{\infty} \frac{(-1)^{r-1}}{r}\left(\sum_{i=0}^{n-1}(n-i) i^{r}\right) z^{r} .
\end{aligned}
$$

Let us recall Stirling's formula:

$$
\log \mathcal{A}\left(\Gamma\left(\frac{1}{z}\right)\right)=-\frac{1}{z} \log z-\frac{1}{z}+\frac{1}{2} \log z+\sum_{r=1}^{\infty} \frac{b_{2 r}}{2 r(2 r-1)} z^{2 r-1}+\text { const }
$$

where $b_{r}$ is the Bernoulli number defined by

$$
\frac{x}{e^{x}-1}=\sum_{r=0}^{\infty} \frac{b_{r}}{r !} x^{r}
$$

We are not interested in the constant term of (4.9) because the asymptotic series in question, (3.5), has no constant term. We can see that substitution of (4.10) in (4.9) eliminates all the logarithmic terms as desired:

$$
\begin{aligned}
& \lim _{m \rightarrow \infty} \log \mathcal{A}\left(\frac{1}{Z_{n}(0)} \int_{\mathcal{H}_{n}} \exp \left(-\sum_{j=2}^{2 m} \frac{(\sqrt{z})^{j-2}}{j} \operatorname{trace}\left(X^{j}\right)\right) d X\right) \\
= & \sum_{r=1}^{\infty} \frac{b_{2 r}}{2 r(2 r-1)} \cdot n \cdot z^{2 r-1}+\sum_{r=1}^{\infty} \frac{(-1)^{r-1}}{r}\left(\sum_{i=0}^{n-1}(n-i) i^{r}\right) z^{r} .
\end{aligned}
$$

Let

$$
\phi_{r}(x)=\sum_{q=0}^{r-1}\left(\begin{array}{l}
r \\
q
\end{array}\right) b_{q} x^{r-q}
$$


denote the Bernoulli polynomial. Then we have

$$
\sum_{i=1}^{n-1} i^{r}=\frac{\phi_{r+1}(n)}{r+1} .
$$

Thus for $r>0$,

$$
\begin{aligned}
\sum_{i=0}^{n-1}(n-i) i^{r} & =\frac{n \phi_{r+1}(n)}{r+1}-\frac{\phi_{r+2}(n)}{r+2} \\
& =\sum_{q=0}^{r} \frac{1}{r+1}\left(\begin{array}{c}
r+1 \\
q
\end{array}\right) b_{q} \cdot n^{r+2-q}-\sum_{q=0}^{r+1} \frac{1}{r+2}\left(\begin{array}{c}
r+2 \\
q
\end{array}\right) b_{q} \cdot n^{r+2-q} \\
& =\sum_{q=0}^{r} \frac{r !(1-q)}{q !(r+2-q) !} b_{q} \cdot n^{r+2-q}-b_{r+1} \cdot n
\end{aligned}
$$

Therefore, we have

$$
\begin{aligned}
& \sum_{r=1}^{\infty} \frac{b_{2 r}}{2 r(2 r-1)} \cdot n \cdot z^{2 r-1}+\sum_{r=1}^{\infty} \frac{(-1)^{r-1}}{r}\left(\sum_{i=0}^{n-1}(n-i) i^{r}\right) z^{r} \\
= & -\sum_{r=1}^{\infty} \frac{1}{2 r} b_{2 r} \cdot n \cdot z^{2 r-1}+\sum_{r=1}^{\infty} \sum_{q=0}^{r}(-1)^{r} \frac{(r-1) !(q-1)}{q !(r+2-q) !} b_{q} \cdot n^{r+2-q} \cdot z^{r} \\
= & -\sum_{r=1}^{\infty} \frac{1}{2 r} b_{2 r} \cdot n \cdot z^{2 r-1}+\sum_{r=1}^{\infty}(-1)^{r-1} \frac{1}{r(r+1)(r+2)} n^{r+2} \cdot z^{r} \\
& +\sum_{r=2}^{\infty} \sum_{q=1}^{[r / 2]}(-1)^{r} \frac{(r-1) !(2 q-1)}{(2 q) !(r+2-2 q) !} b_{2 q} \cdot n^{r+2-2 q} \cdot z^{r} .
\end{aligned}
$$

It is time to switch the summation indices $r$ and $q$ to $g$ and $s$ as in (3.5). The first sum of the third line of (4.11) is the case when we specify a single point on an algebraic curve of arbitrary genus $g=r$. The second sum is for genus 0 case with more than two points specified. So we use $s=r+2$ for the number of points. In the third sum, $q=g \geq 0$ is the genus and $r+2-2 q=s \geq 2$ is the number of points. Thus (4.11) is equal to

$$
\begin{aligned}
& \sum_{g=1}^{\infty} \zeta(1-2 g) \cdot n \cdot z^{2 g-1}+\sum_{s=3}^{\infty}(-1)^{s-1} \frac{1}{s(s-1)(s-2)} n^{s} \cdot z^{s-2} \\
+ & \sum_{g=1}^{\infty} \sum_{s=2}^{\infty}(-1)^{s-1} \frac{(2 g+s-3) !}{(2 g-2) ! s !} \zeta(1-2 g) \cdot n^{s} \cdot z^{-2+2 g+s},
\end{aligned}
$$


where we used Euler's formula

$$
\zeta(1-2 g)=-\frac{b_{2 g}}{2 g}
$$

and the fact that $b_{0}=1$ and $b_{2 q+1}=0$ for $q \geq 1$. Note that the first two summations of (4.12) are actually the special cases of the third summation corresponding to $s=1$ and $g=0$. Thus we have recovered the formula due to Harer-Zagier [2] and Penner [7]:

$$
\begin{aligned}
& \sum_{\substack{g \geq 0, s>0 \\
2-2 g-s<0}}(-1)^{s} \chi\left(\mathfrak{M}_{g, s}\right) n^{s} \cdot(-z)^{-2+2 g+s} \\
= & \lim _{m \rightarrow \infty} \log \mathcal{A}\left(\frac{1}{Z_{n}(0)} \int_{\mathcal{H}_{n}} \exp \left(-\sum_{j=2}^{2 m} \frac{(\sqrt{z})^{j-2}}{j} \operatorname{trace}\left(X^{j}\right)\right) d X\right) \\
= & -\sum_{\substack{g \geq 0, s>0 \\
2-2 g-s<0}} \frac{(2 g+s-3) !}{(2 g-2) ! s !} \zeta(1-2 g) \cdot n^{s} \cdot(-z)^{-2+2 g+s} .
\end{aligned}
$$

\section{REFERENCES}

[1] D. Bessis, C. Itzykson and J. B. Zuber: Quantum field theory techniques in graphical enumeration, Advances in Appl. Math. 1 (1980) 109-157.

[2] J. Harer and D. Zagier: The Euler characteristic of the moduli space of curves, Invent. Math. 85 (1986) 457-485.

[3] M. Kontsevich: Intersection theory on the moduli space of curves and the matrix Airy function, Commun. Math. Phys. 147 (1992) 1-23.

[4] M. L. Mehta: Random Matrices, 2nd Ed., Academic Press (1991).

[5] M. Mulase: Matrix integrals and integrable systems, in topology, Geometry and Field Theory, K. Fukaya et. al. Eds., World Scientific Publ. Co. (1994) 111-127.

[6] M. Mulase: Algebraic theory of the KP equations; in Perspectives in Mathematical Physics, R. Penner and S. T. Yau, Eds., International Press Inc. (1994) 151-217.

[7] R. C. Penner: Perturbation series and the moduli space of Riemann surfaces, J. Differ. Geom. 27 (1988) 35-53.

[8] G. 't Hooft: A planer diagram theory for strong interactions, Nuclear Phys. B72 (1974) 461-473.

[9] E. Witten: Two dimensional gravity and intersection theory on moduli space, Surveys in Diff. Geom. 1 (1991) 243-310. 